\title{
Genitiv und Lativ, Adjektiv und Plural
}

Die meisten obsoleten Kasusendungen der finnischen Sprache sind Lativsuffixe, die zu Partikeln erstarrte Bestimmungen des Ortes oder der Art und Weise bilden. Auf das Primat der Endungselemente verweist auch deren einfache Lautstruktur. Alle, sowohl $n, * k, j$ als auch $s$ enthalten nur ein konsonantisches Phonem. Wie die entfernt verwandten Sprachen bezeugen, sind $n, k$ und $j$ uralt und auch $s$ geht auf die finnischwolgaische Zeit zurück.

Beachtenswert ist, dass speziell $n, k$ und $j$ als äusserst funktionsreiche Suffixe auftreten. Auch wenn man neben $n$ ein mouilliertes $n$ annehmen würde, wäre die Situation nicht viel einfacher. Im Mordwinischen, das diese Doppelheit als einzige Sprache stützt, ist die Verteilung sehr ungleichmässig und spricht keinesfalls für die von Setälä behauptete Gegensätzlichkeit von Possessiv und Lativ. Auch die Aufspaltung der anderen in Frage stehenden Suffixe ist sehr willkürlich. Im folgenden untersuche ich diese homonymen Suffixe einmal allein unter dem morphologischen Aspekt und lasse vorläufig das Problem beiseite, wie Form und Funktion sich zueinander verhalten.

\section{Finnisch.}

1.1. n. Aufgrund der Aussage der Sprachgeschichte können die Akkusativformen $n<* m$ aus den Belegen dieses Suffixes eliminiert werden. Trotzdem hat die Gegenwartssprache wenigstens vier verschiedene $n$-Suffixe ererbt.

1.1.1. Genitiv-Instruktiv. Im Finnischen hat dieser Fall die Aufgabe, zwei Satzglieder miteinander zu verbinden. Ursprünglich ist der $n$-Kasus stets die Form der Bestimmung, obgleich die in den Postpositions- und Nominalkonstruktionen eingetretene Entwicklung zu einer teilweisen Unabhängigkeit dieses 
Falles geführt hat. Falls der $n$-Kasus als Sonderbestimmung des Nomens auftritt, handelt es sich um einen Genitiv (Possessiv), während die das Verb bestimmende $n$-Form wiederum ein Instruktiv ist. Beide stehen in der Funktion einer freien Bestimmung: d.h. sie können wegbleiben, ohne dass die Satzkonstruktion dadurch zerstört wird. Im Neufinnischen sind Genitiv und Instruktiv formal verschiedene Kasus, aufgrund ihrer unterschiedlichen Pluralformen.

1.1.2. Lativ. Zweifellos ist das lativische $-n$ mit den Endungen des Illativs und Allativs verbunden; ferner lassen sich diverse Partikeln aus der Zeit von Agricola anführen, wie taan und tyjön. Im übrigen ist es sehr schwer, eine Grenze zu ziehen zwischen dem lativischen bzw. adverbalen Genitiv und dem normalen adnominalen Genitiv, vgl. Puhujan menivät paperit sekaisin 'Dem Redner gingen die Papiere durcheinander' und Puhujan paperit menivät sekaisin 'Die Papiere des Redners gingen durcheinander' oder Naapurin vietiin auto pihalta 'Dem Nachbarn wurde das Auto vom Hof gestohlen' und Naapurin auto vietiin pihalta 'Das Auto des Nachbarn wurde vom Hof gestohlen'. Der »adverbale» Genitiv ist hier nur eine von ihrem Bezugswort getrennte, selbständig werdende adnominale Bestimmung, die gleichzeitig die einem possessiven Richtungskasus eigene Funktion erhalten hat.

1.1.3. Adjektiv. Ravila erwähnt aus dem Estnischen adjektivische Genitive wie mullatükk (FUF XXVII 76), doch findet man auch im Finnischen entsprechendes: porsaankyljys 'Schweinskotelett' (Kotelett vom Schwein) oder kullanpala 'Goldstück' (Stück aus od. von Gold). Die sog. Definitiv- oder Substanzgenitive sind in ihrer Gesamtheit adjektivisch, da die Bestimmung in ihnen begrifflich das Bezugswort deckt.

1.1.4. Das obsolete Pluralzeichen $n$ begegnet in einigen Possessivsuffixen als Erinnerung an die Zeiten, da das Sprachsystem neben der Pluralität des Besitzers auch die Pluralität des Besitzes notierte. Ohne weiteres denkt man auch an die in zahlreichen Sprachen vorhandenen, auf $n$ - anlautenden pluralischen Demonstrativpronomina nämä, nuo, ne. Ist es nur ein Zufall, dass der Träger ihrer Pluralität ein als Präfix zu analysierendes $n$ ist? 
Aus dem oben Ausgeführten geht hervor, dass die possessivische, lativische und adjektivische Funktion der -n-Form auch hinsichtlich des Neufinnischen miteinander in Beziehung zu setzen ist. Die Pluralfunktion dagegen bleibt losgelöst.

1.2. $k$. Das $-k$ im Wortauslaut lebt in den meisten finnischen Dialekten als Verursacher der Auslautdoppelung, in einzelnen Savo-Mundarten sogar als Palatalklusil, hörbar vor der Pause und vor dem Vokal. Die Schriftsprache verzeichnet den Laut nicht; ein sichtbarer Beweis aber für die magische Wirkung des $-k$ ist die schwache Stufe der diesbezüglichen Form, falls darin ein Konsonant oder eine Konsonantenverbindung vorkommt, die dem Stufenwechsel untersteht.

1.2.1. Possessive Funktion hat diese Form im Finnischen nicht und scheint sie auch nie gehabt zu haben. Eine instruktivische Funktion lässt sich dagegen feststellen, oft in zusammengesetzten Ausdrücken, die auf einem -n-Instruktiv aufbauen: kah(d)areisin.

1.2.2. In der finnischen Sprache hat die $k$-Form vor allem eine lativische Funktion. Insgeheim auf $-k$ auslautende Formen sind als Richtung angebende Partikeln relativ häufig: taa $<*$ tayak, luo $<*$ lōk, alemma $<*$ alempak usw. Ferner ist der k-Lativ die historische Kasusform des 1. Infinitivs: antaa $<*$ antadak, und begegnet als Bestandteil in zahlreichen zusammengesetzten Suffixen: im Translativ $k+s$, im Prolativ $c+k$, im Terminativ $n n+k$, im Abessiv $t t a+k$.

1.2.3. Als eigentliches Adjektivsuffix begegnet $k$ nicht, ist aber sonst ein äusserst produktives sowohl denominales als auch deverbales Nominalsuffix. Verwandt mit der letztgenannten Funktion ist bekanntlich sein Auftreten als Präsenszeichen.

1.2.4. Das unsichtbare $-k$ bedeutet die Pluralität der Personalpronomina und gelangte auf diesem Wege zur Bezeichnung der Mehrheit des Besitzers in die Personalendungen der Verbalflexion und in die Possessivsuffixe.

Vom Standpunkt des Neufinnischen lassen sich die einzelnen Funktionen des $k$-Suffixes kaum miteinander verbinden, doch ist beachtenswert, dass es auch im Neufinnischen zwei Funktionen mit dem $n$-Suffix gemeinsam hat: die Lativität und die Pluralität. 
1.3.1. Eine possessive Kasusfunktion besitzt das Suffix $j$ nicht, einige Formen könnten jedoch ebenso gut als modalinstruktivisch wie adjektivisch erklärt werden, z.B. avo(i)silmin, vgl. kahareisin.

1.3.2. Solche Formen wie irti, julki, ilmi, auki werden als alte $i$-Lative gedeutet.

1.3.4. Als Adjektivableitung erscheint $j(i)$ z.B. in den Komposita aitovieri, lehmisavu, huhtikuu, Koirikivi. Sonst ist das $i$ ja sehr üblich sowohl als denominales wie auch als deverbales Nominalsuffix.

1.3.4. In den obliquen Fällen des Plurals - mit Ausnahme des zweiten Genitivs - begegnet $i \sim j$ als Pluralcharakter.

Im Finnischen erscheinen alle die oben behandelten Suffixe als Instruktive - als freie Bestimmungen des Verbs - , als Lative und Pluralcharakter. Würde die Funktion stets ein eigenes Morphem voraussetzen, kämen hier neun verschiedene Morpheme in Frage, drei für eine jede Funktion! Das zehnte wäre der possessive Genitiv, das elfte der Dativgenitiv, und von den Ableitungssuffixen würden zumindest auf $k$ und $j$ je zwei oder drei entfallen, insgesamt wären es also an fünfzehn Suffixe!

\section{Lappisch.}

2.1. $n$. Das $-n$ im Wortauslaut ist in den meisten lappischen Dialekten geschwunden, es kann aber aufgrund einiger Pronomina und des Südlappischen auf die diesbezüglichen Formen zurückgeführt werden.

2.1.1. Possessiv-Instruktiv. Im Singular lautete dieser Kasus auf $-n$ aus, z.B. (Røros) vietlan bieńa 'der Hund des Bruders', duonan mana 'roh und böse (wörtl. des Teufels Kind)'. Instruktivische Nuancen sind sehr üblich: čârmə-gietan 'mit geballten Fäusten', iehkəd’ən 'am Abend', ganz zu schweigen vom Instruktiv-Genitiv des Verbalstammes: $\operatorname{lpN}$ bōđii čuoigâ 'kam auf Skiern'.

2.1.2. Lativ. Das Attribut der pluralischen Lokalfälle steht im Lappischen im Lativ (oder ebenso gut im Genitiv). Es wird angenommen, dass sich der Kasus erst in Verbindung mit einem illativischen Bezugswort stabilisiert hat und von da aus in die 
übrigen Stellungen gelangt ist (Itkonen, Congressus secundus internationalis Finno-Ugristarum Pars I 205). Ferner begegnet der $n$-Lativ in einigen Partikeln, die die Richtung angeben: Røros čâkičz duøppələn 'gegen den Spätherbst' und als Endungsphonem des Illativs: (Wiklund) Gardenāsan 'ins Haus', seelapp. dahkkujen 'dem Werke'.

2.1.3. Adjektiv. Einen eigentlichen mit $n$ abgeleiteten Derivationstypus kennt das Lappische nicht, obgleich der Genitiv in einigen Verbindungen wie im Finnischen als adjektivisch aufgefasst werden kann: Røros riewdien nierə 'eiserner Mann'.

2.1.4. In den lappischen Possessivsuffixen begegnet dasselbe

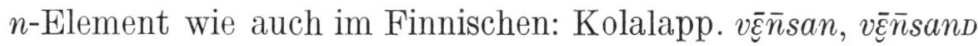

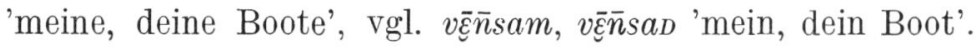

Insgesamt gesehen sind die Funktionen des $n$-Suffixes im Lappischen recht ähnlich wie im Finnischen, auch was ihre Ausdehnung angeht.

2.2. $k$. Begegnet im Lappischen als $k$ oder $k A$, mitunter geschwunden.

2.2.1. Für die Possessivfunktion liegen keine Belege vor. Als freie Verbalbestimmung tritt die $k$-Form dagegen auf: Røros čâkča duoka 'gegen den Herbst', lpN bæi'veg 'am Tag'.

2.2.2. Die lativische Funktion begegnet vor allem in einigen Pronomina: lpN dâggŏ 'dorthin', âlâ <*ülek 'über'.

2.2.3. Als Adjektivableitung tritt das $k$-Suffix nicht eigentlich auf, wohl aber als allgemeine Nominalableitung: bēenâ bèednâgâ 'Hund: des Hundes'.

2.2.4. Der ursprüngliche $k$-Plural dürfte im Lappischen nicht begegnen (Ravila, FUF XXVII 67, 72).

2.3. $j$. Abhängig von der Silbenstellung $j$ oder $i$, wie auch im Finnischen.

2.3.1. Genitiv. Der Gen.Pl. im Lappischen ist - wie man sagt - der endungslose Pluralstamm. An sich ist jedoch gerade $j$ die Endung, obwohl es als Pluralzeichen und nicht als Kasusendung ausgelegt wird. (Vgl. das Estn., wo die pluralischen obliquen Kasus ebenfalls auf der Basis des Gen.Pl. gebildet werden: härgade:härgadele usw. 'der Ochsen : den Ochsen'.) Z.B. Røros manaj v́ätna 'die Arbeit der Kinder'. 
2.3.2. Das lativische $j$ begegnet wenigstens in einigen Partikeln: IpN duokkai 'nach hinten', vuollai 'unter'.

2.3.3. Das Adjektivsuffix $j$ ist auch dem Lappischen bekannt, z.B. guollai 'fischreich' (guolle 'Fisch'). Ravila hält den Gen.Pl. des Lapp. speziell für eine adjektivische Ableitung.

2.3.4. Wie auch im Finnischen sind die pluralischen obliquen Fälle des Lappischen auf das Suffix $j$ angewiesen. Im Lichte des Obengesagten wirken die $j$-Formen jedoch eigenartig heterogen. Das $j$ des Plural hat sich schon als Genitivendung und Adjektivableitungssuffix erklärt. Toivonen hat auf die Möglichkeit hingewiesen, dass das $j$ in den pluralischen Illativformen lativisch sei. Im Illat.Sg. dahkkujen sind zwei Lativphoneme verbunden, $j$ und $n$, wie im Illat.Sg. Gardenāsan das $s$ und $n$ (FUF XXVIII 14). Im letzteren Fall ist es konsequent, das lativische $j$ auch in die übrigen Lokalkasus zu bringen. Dann wird der Inessiv Sg. güliin 'in den Fischen' hinsichtlich seines Endungselements erklärt als entstanden auf der Grundlage des $j$ des Lativs und des na des Lokativs, also $* k a l a+j+$ $n a$, so wie das lativische $s$ und der Lokativ dem Inessiv Sg. zugrundeliegen: Røros âktəña bielasn 'auf einer Seite'. Ferner kann auch das $j$ des Genitivs vor diesem Hintergrund ebenso gut als lativisches wie als possessivisches, pluralisches oder adjektivisches Element erklärt werden.

Im lapp. $j$-Plural scheinen sich alle möglichen Funktionen des Suffixes $j$ zusammengefunden zu haben, die in der Gegenwartssprache jedoch die gemeinsame Aufgabe haben, die Pluralität auszudrücken. Weist das nicht darauf hin, dass es unbegründet wäre, die $j$-Suffixe jeweils von einer eigenen eigenartigen homonymen Urform herzuleiten? Offenbar war das $j$-Element von Anfang an das Zeichen eines syntaktischen untergeordneten Verhältnisses und seine vielfältigen, aber unbestimmten Funktionen differenzierten sich später.

3. Mordwinisch.

3.1. Das Suffix $n$. Das Mordwinische ist hinsichtlich dieses Suffixes problematisch, da das $\dot{n}$ dort überwiegend mouilliert 
auftritt. An sich vertritt es also sein eigenes Morphem, fällt aber hinsichtlich der Distribution mit dem $n$-Element der übrigen Sprachen zusammen, so dass es angebracht sein dürfte, es in diesem Zusammenhang zu behandeln.

3.1.1. Genitiv. Der mordwin. ń-Kasus hat alle Funktionen des finn. $n$-Kasus, sogar in betonter Weise. Possessivisch ist er äusserst häufig: (Erza) tejtéreń ṕra 'der Kopf des Mädchens', ajgoron griva 'die Mähne des Pferdes', desgleichen in habeoKonstruktionen: at'ań babań a ejdest a kakšost 'Vater und Mutter haben weder Sohn noch Tochter'. Er begegnet als Agens der Nominalform vom Verb: tetan sakavt moda 'das vom Grossvater gepflügte Feld', ja sogar als deutliche "adverbale» Bestimmung: A erävi monén toń platiat 'Ich brauche deinen Mantel nicht' (Feoktistov) oder - in Verbindung mit einem transitiven Verb: kodamo paro tejat ton monen' 'Was du mir auch Gutes tust' (Paasonen). Der instruktivische Gebrauch ist auch ausserordentlich ausgedehnt: kavton' 'zu zweit', vejkeń 'allein'.

3.1.2. Lativ. Eindeutig lativisch begegnet die $n$-Form in den Pronominalausdrücken kozoń 'wohin', tozoń 'dorthin', ferner ist sie in der Allativendung enthalten: modane(ń) 'aufs Feld'.

3.1.3. Als Adjektiv ist die n-Form äusserst allgemein: ošoń lomań 'Städter' (Stadtmensch), (Mokscha) t'alzń iläd 'WinterAbend'.

3.1.4. In der possessiven Flexion erscheint als Pluralzeichen ein nichtmouilliertes $n$ : t'soram 'mein Sohn': t'soran 'meine Söhne' oder in der 3. Sg. t'śorazo od. t'śoranzo für die Pluralität des Besitzes.

Der letztgenannte Punkt weist darauf hin, dass das pluralische $n$ der Possessivsuffixe selben Ursprungs ist wie die entsprechenden Morpheme im Finnischen und Lappischen; das in den sonstigen Fällen auftretende ń-Element enthält zwei Morpheme: $n$ und die Palatalisation. Dialektweise kann im Erza das nichtmouillierte $n$ in solchen Aufgaben auftreten wie z.B. louun lomań (Schriftsprache lovoń lomań) 'Schneemann' (Serebrennikov, Istoritšeskaja morfologija mordovskich jazykov 16), so dass das mouillierte $n$ des Mordwin. als Ergeb- 
nis einer späten Entwicklung erklärt werden könnte. Man kann auch feststellen, dass die Palatalisation die Funktion des Suffixes nicht ändert: das finn. nichtpalatalisierte $n$ hat genau dieselbe Funktion wie das mordwin. palatalisierte (und entspricht natürlich ausserdem dem nichtpalatalisierten $n$ der mordwin. possessiven Flexion). Im mordwin. $\dot{n}$ hat man Spuren des $\eta^{\prime}\left(c^{\prime}\right)$ gesucht und es mit dem finn. Ableitungssuffix ise verglichen. Allgemein erscheint ja ${ }^{*} \dot{\eta}$ im Mordwin. als $j$. In irgendeinem Zwischenstadium hätte $\dot{\eta}+n$ auf jeden Fall das Resultat $j+n$ ergeben. Es wäre wohl ebenso gut möglich, dass im mordwin. $n$ ein primäres $j$ mit $n$ verbunden enthalten wäre, wie es im Illat. Pl. des Lappischen der Fall ist. Falls das mordwin. $n$ in zwei Bestandteile zerlegt werden kann, haben beide genau dieselbe Funktion gehabt.

3.2. $k$. Auf ein blosses $-k$ ausgehende Formen gibt es im Mordwinischen nicht; das entsprechende Suffix erhält die Form $k_{A}$. Ein intervokalisches $k$ hat sich nicht erhalten, das Ergebnis war das Suffix va.

3.2.1. Für die possessive Verwendung liegen keine Belege vor.

3.2.2. Die lativische Funktion erscheint in den Pronominalformen tuva 'dorthin', kuva 'wohin'. Im heutigen Kasussystem begegnet der *ks-Lativ als Prolativ: kiava 'den Pfad entlang' und im Endungselement des Translativs: modaks 'zu Acker'.

3.2.3. Als Adjektivableitungssuffix kommt $* k$ nicht in Frage, möglicherweise ist es in einigen allgemeinen Nominalableitungssilben verborgen.

3.2.4. Auch die Funktion der Pluralität scheint das Suffix $k$ im Mordwinischen nicht zu haben.

3.3.j. Dieses Suffix scheint sowohl als Kasus- wie auch als Suffixelement im Mordwinischen völlig zu fehlen. Collinder sagt z.B., das $j$ des Plural sei geschwunden, so dass das Mordwin. keine unbestimmte Kasusflexion im Plural habe. Der vollständige Schwund könnte sich auch so erklären, dass $j$, wie oben angedeutet, mit dem $n$-Suffix verschmolzen ist, wodurch es über alle in Frage kommenden Funktionen verfügt. Andererseits ist es überhaupt nicht gesagt, dass $j$ im Mordwin. der Pluralcharakter gewesen sein muss (vgl. das Tscheremissische). 


\section{Tscheremissisch.}

4.1. n. Im Tscheremissischen gibt es einen ursprünglich nur für belebte Begriffe geltenden possessiven Genitiv, der auch als Konjunktwort in Postpositionskonstruktionen und als Agens von Nominalformen erscheint. Ferner begegnet er als indirekter adnominaler Kasus in habeo-Konstruktionen und in der Funktion des indirekten Objekts neben dem transitiven Verb (Kangasmaa-Minn, MSFOu 146 43-46). Die instruktivische, modale und temporale Verwendung des Kasus entspricht genau jener der diesbezüglichen Formen des Lappischen und Mordwinischen oder des finnischen Instruktivkasus. $\mathrm{Zu}$ beachten ist, dass mit dem adverbalen Genitiv keine Kriterien der Person verbunden sind. Bei der halbadverbalen bzw. dativischen Verwendung des Genitivs tritt diese Eigenschaft dagegen ganz deutlich hervor.

4.1.2. Lativ. Der lativische $n$-Kasus tritt nicht rein auf, sondern als Suffix an. Itkonen führt den Lautunterschied auf eine Veränderung der Druckverhältnisse zurück und führt

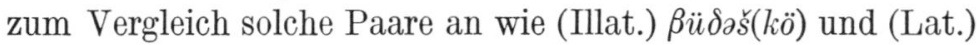
$\beta \ddot{u} \delta e s ̌$ 'ins Wasser' oder die Gerundien der em- und am-Verben kajen 'im Gehen' und tolan 'im Kommen' (FUF XXIX 238). Auf jeden Fall ist die an-Form, die sich in Pronomina und Postpositionen kristallisiert hat, eindeutig lativisch und entspricht dem eš-Kasus der Normalflexion.

4.1.3. Adjektivsuffix. Man hat im tscheremiss. Genitiv eine Adjektivableitung sehen wollen und ihn mit dem mordwin. Genitiv verglichen. Die Zusammenstellung hinkt jedoch. Die wenigen Genitivformen, die im Tscheremissischen in der Funktion des Adjektivs auftreten können, sind nie possessiv, sondern in erster Linie instruktiv in ihrer Bedeutung. Ausserdem bezeichnen sie alle unbelebte Begriffe, und gerade im Tscheremiss. beschränkt sich der adnominale Genitiv fast ausschliesslich auf belebte. Die an-Possessivableitung ist dagegen in der Sprache reichlich vorhanden: imñan 'mit Pferd versehen', vgl. imńan 'mit dem Pferd' oder 'des Pferdes'. Mit anderen Worten: den mordwin. ń-Formen entsprechen im Tscheremiss. einmal die mit dem $n$-, zum andern die mit dem an-Suffix versehenen Konstruktionen. Der adnominale Genitiv begegnet sehr selten, 
und der überwiegend grösste Teil der an-Ableitungen beruht auf Nomina, die - weil sie unbelebt sind - nie im Genitiv auftreten würden. Deshalb ist es nicht recht glaubhaft, dass das an-Possessivadjektiv aus dem Genitiv lediglich als Resultat der Druckverschiebung entstanden sein soll. Itkonen ist der Ansicht, das $a$ der am-Verben verberge das reflexive Suffix $v$ in sich (MSFOu 125 106). Weshalb könnte das Suffix an dann nicht aus zwei Suffixen bestehen, $j+n$, und somit den mordwin. $\dot{n}$-Formen vergleichbar sein?

4.1.4. Plural. Der formale Plural ist im Tscheremissischen schwach entwickelt und meist auf Lehnelemente angewiesen. Für keins der in Frage stehenden Suffixe kann im Tscheremiss. die Funktion der Pluralität nachgewiesen werden.

4.2. k. Für dieses Suffix kann keine possessive oder pluralische Verwendung nachgewiesen werden. Die Sprache verfügt zwar über begrenzte Nominalsuffixe mit $k$, doch können mit deren Hilfe keine Possessivadjektive entstehen. So bleibt für das $k$ im Tscheremiss. nur eine Funktion.

4.2.2. Lativ. Eigentlich müsste diese Form Illativ genannt werden, denn als Bestandteil von Postpositionskonstruktionen entspricht sie genau dem Illativ der normalen Nomina: bake 'auf', dek(e) 'hin, zu'.

4.3. $j$. Itkonen meint, der $j$-Lativ sei verborgen in Apposi-

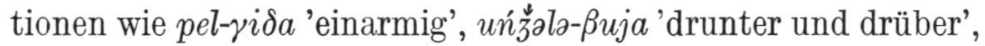
wo er mit dem Füllvokal zu $a$ verschmolzen sei, also offensichtlich $k i t+a+j$ und $\beta u j+a+j$ (Itkonen, Virittäjä 1967 254-255). Dies stützt meine obige Vermutung, wonach auch die Formen mit einem an-Suffix ein $j$-Element enthalten: kit $+\imath+j+n$. Dann ist es im Prinzip gleichgültig, ob am Ende der Form $\beta u j a$ das $j$ oder das $n$ oder beide geschwunden sind; der Vollvokal $a$ setzt schon als solcher ein $j$ voraus. Im übrigen ist bemerkenswert, dass das Tscheremiss. wie das Mordwin. das Suffix $j$ in keiner in der Gegenwartssprache sichtbaren Funktion kennt.

5. Permische Sprachen. Je weiter wir uns vom Ostseefinnischen entfernen, desto unbestimmter scheinen die Funktionen der Suffixe $n, k$ und $j$ zu werden. Der »bezeichnete» Genitiv als adnominaler Kasus verschwindet von der Bildfläche; anderer- 
seits könnte man natürlich beispielsweise den Adessiv der permischen Sprachen und den Dativ des Ungarischen aufgrund der Funktion als Genitive bezeichnen.

5.1. Der adnominale $n$-Kasus tritt also in den permischen Sprachen nicht auf, wohl aber der adverbale. Instruktivformen wie der syrjän. Instrumental kiken 'zu zweit', ojęn 'bei Nacht' oder die entsprechenden wotjakischen Kasusformen ogen, kiken 'allein, zu zweit' schliessen sich semantisch einwandfrei den "Instruktiven" der finnisch-wolgaischen Sprachen an. Ebenso gut können sie aber mit den Lokativen der ugrischen Sprachen verglichen werden. Es sieht so aus, als hätte eine Sprache ohne possessiven Genitiv auch keinen adverbalen.

5.1.2. Lativ. Die n-Adverben des Wotjakischen sind als Lative erklärt worden: kimin 'umgestülpt', t'škin 'kopfüber'. Diese sind zu vergleichen mit den tscher. Formen tuрәń 'mit dem Rücken', meləń 'mit der Vorderseite', die vom Standpunkt der letzteren Sprache keine reinen $n$-Lative sein können (im Tscheremiss. ist auch das »lativische» $n$ nicht mouilliert).

5.2. $k$. Das $k$ im Wortauslaut ist in den permischen Sprachen geschwunden, verbirgt sich jedoch noch hinter manch vokalisch auslautendem Kasus.

5.2.2. Das ist der syrjän. Allativ, Illativ, Transitiv und die entsprechenden Kasus im Wotjakischen: (syrj.) ul-e 'hinunter'.

5.3.j. Dieses Suffix ist in den permischen Sprachen kaum sichtbar.

5.3.2. Es soll jedoch lativisch in den syrjän. Prolativen seti 'dort(hin), jenen Weg' tati 'hier(hin), diesen Weg' erscheinen; hinsichtlich der Bedeutung könnten die Formen ebenso gut Instruktive sein.

5.3.3. Das Suffix $j$ begegnet im Wotjakischen deminutiv: pužei 'kleines Rentier', desgleichen adjektivisch: umoi 'gut'. Aus dem Syrjänischen liegt der Beleg kenei 'Auerhenne' vor.

6. Obugrische Sprachen.

6. 1. n. Der ugrische Zweig kennt offenbar überhaupt keinen $n$-Kasus. Die "instruktivischen» $n$-Formen sind dort ursprüngliche Lokative (Liimola, MSFOu 127 154-155).

6.1.4. Im Wogulischen wird die Mehrheit des Besitzes durch 
ein im Possessivsuffix infixiertes $n$ ausgedrückt: haapumt 'in meinem Boot', haapanumt 'in meinen Booten'.

6. 2. $k$. In den ugrischen Sprachen ist der $k$-Lativ deutlich beliebter als die anderen Lativtypen.

6.2.2. Das alte $-k(-\gamma,-g,-h)$ erscheint in der Endung des Translativ-Essivs und in einzelnen Partikelformen: wog. ti 'hierher', südostjak. tǒh 'dorthin', wog. tau jängyg jenitys 'er wuchs lang (= er wurde gross)'.

\section{3. $j$.}

6.3.2. Toivonen sieht einen $j$-Lativ in Formen wie ostj.

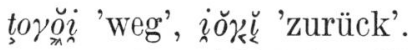

6.3.3. Das Adjektivsuffix $j$ begegnet sowohl im Wogulischen als auch im Ostjakischen: ostj. xònì 'zum Haus gehörig', wog. täli 'winterlich'.

7. Ungarisch.

7.1. n. Falls ungar. szépen 'schön (adv.)', ketten 'zu zweit' vom Lokativ und nicht vom »Instruktiv» abgeleitet wird, kann man feststellen, dass das Suffix $n$ im Ungarischen völlig fehlt. Es begegnet dort auch nicht in der possessiven Flexion.

7.2. $k$.

7.2.2. Lativ. Ein geschwundenes $k$ verbirgt sich in den ungarischen Wohin-Fällen: hová 'wohin'; ein erhaltenes $k$ erscheint in der Dativendung: embernek 'dem Menschen'.

7.3.j.

7.3.2. Toivonen suchte im Ungarischen nach dem $j$-Lativ. Nach Hajdú hat sich im Ungarischen folgende Entwicklung vollzogen: fiu. $* k>$ protoungar. $\gamma>u / \ddot{u}, j$, so dass er auch alle auf $j$ hinweisenden Formen von $k$ ableitet (Bevezetés az uráli nyelvtudományba 125).

7.3.3. Das Adjektivableitungssuffix $j$ ist im Ungarischen recht produktiv: tegnapi 'gestrig', isteni 'göttlich'.

7.3.4. Plural. Das pluralische $j$ erscheint in der possessiven Flexion, wo es die Mehrheit des Besitzes ausdrückt: szobám 'mein Zimmer', szobáim 'meine Zimmer'.

Aus den obigen Ausführungen geht hervor, dass die Belege von $n$ und $j$ (sowie die der aus ihnen gebildeten, angenommenen zusammengesetzten Suffixe) in den in Frage kommenden Spra- 


\section{Zusammenfassung}

Posses- Instruk- Lativ Adjek- Plural

1. $n$ siv tiv tiv

Finnisch

Lappisch

Mordwinisch

Tscheremissisch

Perm. Spr.

Obugr. Spr.

$\begin{array}{ccccc}\times & x & \times & x & \times \\ \times & x & x & \times & \times \\ & & & & x\end{array}$

$n+j$

Mordwinisch

Tscheremissisch

Lappisch

$x \quad x$

$\times(?)$

$x$
$x$

2. $k$

Finnisch

Lappisch

Mordwinisch

Tscheremissisch

Perm. Spr.

Obugr. Spr.

Ungarisch

$3 \cdot j$

Finnisch

Lappisch

Mordwinisch

Tscheremissisch

Perm. Spr.

Obugr. Spr.

Ungarisch

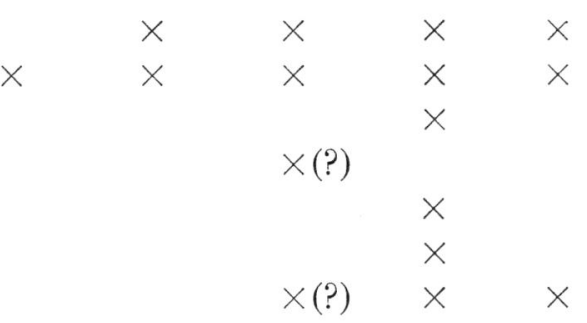

chen unter den einzelnen Funktionen recht gleichmässig verteilt sind. Die ursprüngliche Funktion lässt sich nicht bestimmen; sowohl $j$ als auch $n$ vertreten ein altes, nicht differenziertes, subordiniertes Element. Eine Sonderstellung behält das plura- 
lische $n$ der Possessivsuffixe, da es auch in die obugrischen Sprachen gelangt ist, wo die anderen Funktionen des $n$ unbekannt sind. Die Belege des dritten Suffixes $k$ konzentrieren sich auf die Funktion des Richtungskasus, die einzige Abweichung in der Tabelle ist das pluralische $k$ im Finnischen (die "instruktivischen" Bedeutungen lassen sich leicht mit den lativischen zusammenstellen). Andererseits könnten wiederum die auf $-k$ ausgehenden, zur finiten Flexion übergegangenen Nominalableitungen der Verben die Brücke zwischen diesen Funktionen bilden. Interessant ist die Beobachtung, dass zwischen ${ }^{*} k$ und ${ }^{*} j$ eine nahezu ergänzende Aufteilung herrscht: $k$ kommt die Lativfunktion $\mathrm{zu}, j$ die possessivische, adjektivische und pluralische Funktion. Auch die gegenseitige lautliche Nähe von $k$ und $j$ (wie auch die des als Zwischenstufe auftretenden $\gamma$ ) legt die Vermutung nahe, dass die genannten Suffixe ursprünglich Varianten eines einzigen morphologischen Elements darstellen.

Eeva KangasmaA-Minn 